\title{
EL ENVEJECIMIENTO DE LA POBLACIÓN EN ARAGÓN
}

\author{
POR \\ M. ${ }^{a}$ JOSÉ AGUILERA ARILLA
}

\section{Introducción}

El envejecimiento de la población mundial es un hecho constatado, gracias a la abundancia de investigaciones en torno a este importante tema de estudio. La prolongación de la vida humana, lejos de constituir un problema, es un logro de la humanidad, consecuencia de las mejoras socioeconómicas y por tanto sanitarias a escala mundial. Si bien siempre han existido personas longevas, que han alcanzado edades mucho más avanzadas que la media de edad esperada para sus conciudadanos, hace relativamente poco tiempo que esa mayor longevidad es alcanzada por un grupo de población proporcionalmente más numeroso. Por eso, traerlo a colación como uno de los problemas del mundo rural puede ser de no muy fácil comprensión a primera vista. Pero las últimas etapas de la vida biológica llevan consigo hoy en día la merma de algunas de las funciones más apreciadas por el conjunto social. Es decir, el envejecimiento demográfico, considerado sólo en su aspecto biológico, es algo apreciado y muy valorado a nivel individual; sin embargo, las consecuencias sociales de ese envejecimiento en una sociedad acarrea problemas para los que hay

M. ${ }^{a}$ José Aguilera Arilla. Departamento de Geografía de la Universidad Nacional de Educación a Distancia, Madrid.

Estudios Geográficos

Tomo LVII, n. ${ }^{\circ} 225$, octubre-diciembre 1996 
que dar soluciones o quizás no dejar que esa sociedad llegue a niveles de mayor envejecimiento, contraponiendo un importante porcentaje de población joven que la dinamice, gracias a la aplicación de la adecuada política demográfica. El problema no es la mayor longevidad de la población, sino el aumento de la proporción de personas de más de 65 años.

\section{El envejecimiento de la población a escala planetaria}

En la actualidad, cuando se confirma en muchos aspectos -económico, electromagnético o de comunicaciones, de redes de información, etc.- la idea de que el mundo se asemeja en su funcionamiento al de una «aldea global» enunciada por MacLuhan en 1962, son muchos y muy variados los aspectos que se distribuyen de forma muy contrastada en ese espacio global. Uno de ellos es precisamente el envejecimiento de la población.

Mientras que en 1985, a escala mundial, el porcentaje de menores de 15 años era de 33,5\%, en 1995 era de $32 \%$, y para los mayores de 65 años, esa cifra se elevaba a $6 \%$ en el primer año y también a $6 \%$, en el segundo. El índice de envejecimiento es, por tanto, mayor en $1995(0,18 / 0,19$ respectivamente). De ahí se puede deducir la cada vez más importante incidencia de la falta de nacimientos. La evolución que expresan estas cifras es bastante significativa en si misma, a pesar de constituir un promedio de otras muy contrastadas para los distintos continentes y paises En primer lugar se observa que, a escala planetaria, el envejecimiento se debe más a una reducción en la proporción de jóvenes, consecuencia de la disminución de la tasa de natalidad, que al aumento del número de personas que sobrepasan la edad de 65 años. Esta misma razón se puede aducir en el estudio individualizado de cada país en la evolución de su historia demográfica.

Sin embargo, si estudiamos comparativamente el espacio mundial en un mismo momento, podemos comprobar que las distintas relaciones de envejecimiento obedecen a proporciones muy diferentes de jóvenes y viejos. Así por ejemplo, si comparamos Norteamérica (USA y Canadá) con Europa, las cifras se asemejan bastante. El porcentaje 
de personas de más de 65 años es exacto en ambos conjuntos espaciales (13\%). Sin embargo, el de jóvenes, en el primero, es de $22 \%$ y en el segundo de $20 \%$ según datos de 1995. Ello nos está indicando el mayor envejecimiento del continente europeo (0,65 frente 0,59 en Norteamérica). Los contrastes son todavía mayores si comparamos estas cifras con las del conjunto latinoamericano, que eran, para la misma fecha, un $5 \%$ para los mayores de 65 años y un $34 \%$ para los de menos de 15 años. Estas cifras nos dan un índice de envejecimiento del 0,15 que indica la gran juventud de este gran espacio subcontinental.

\section{CUADRo I}

PORCENTAJE DE JOVENES Y VIEJOS E ÍNDICE DE ENVEJECIMIENTO DE LOS PAISES AMERICANOS (AÑO 1995)

\begin{tabular}{|c|c|c|c|c|c|c|c|}
\hline Países & $\begin{array}{c}\% \\
\text { menos } \\
\text { de } 15 \\
\text { años } \\
J\end{array}$ & $\begin{array}{c}\% \\
\text { más } \\
\text { de } 65 \\
\text { años } \\
V\end{array}$ & $\begin{array}{c}\text { Índice } \\
\text { de } \\
\text { envejeci- } \\
\text { miento } \\
\mathrm{V} / \mathbf{J}\end{array}$ & Países & $\begin{array}{c}\% \\
\text { menos } \\
\text { de } 15 \\
\text { años } \\
J\end{array}$ & $\begin{array}{c}\% \\
\text { más } \\
\text { de } 65 \\
\text { años } \\
V\end{array}$ & $\begin{array}{c}\text { Índice } \\
\text { de } \\
\text { envejeci- } \\
\text { miento } \\
\mathbf{V} / \mathbf{J}\end{array}$ \\
\hline América del $N . .$. & 22 & 13 & 0,59 & Méjico & 36 & 4 & 0,11 \\
\hline EE. UU. ... & 22 & 13 & 0,59 & Nicaragua . & 46 & 3 & 0,07 \\
\hline Canadá ........... & 21 & 12 & 0,57 & Panamá .......... & 34 & 5 & 0,15 \\
\hline Lat./Car. ......... & 34 & 5 & 0,15 & Ant. y Barb. ...... & 25 & 6 & 0,24 \\
\hline Argentina ........ & 30 & 9 & 0,30 & Bahamas ......... & 29 & 5 & 0,17 \\
\hline Bolivia ............ & 41 & 4 & 0,10 & Barbados ......... & 24 & 12 & 0,50 \\
\hline Brasil ............. & 32 & 5 & 0,16 & Cuba ............... & 22 & 6 & 0,27 \\
\hline Chile .............. & 31 & 6 & 0,19 & Dominica ......... & 29 & 8 & 0,28 \\
\hline Colombia ......... & 33 & 5 & 0,15 & R. Dominicana .. & 35 & 4 & 0,11 \\
\hline Ecuador .......... & 38 & 4 & 0,10 & Granada .......... & 43 & 5 & 0,12 \\
\hline Guyana ........... & 32 & 4 & 0,13 & Guadalupe ....... & 26 & 8 & 0,31 \\
\hline Paraguay ......... & 40 & 4 & 0,10 & Haiti ........ & 40 & 4 & 0,10 \\
\hline Perú ............... & 36 & 4 & 0,11 & Jamaica .......... & 33 & 8 & 0,24 \\
\hline Suriname ........ & 35 & 5 & 0,14 & Martinica ........ & 23 & 10 & 0,43 \\
\hline Uruguay .......... & 26 & 12 & 0,46 & Antillas H. ....... & 26 & 7 & 0,27 \\
\hline Belize ........... & 44 & 4 & 0,09 & Puerto Rico ...... & 27 & 10 & 0,37 \\
\hline Costa Rica ....... & 35 & 5 & 0,14 & Stukitts-N. ....... & 32 & 9 & 0,28 \\
\hline Salvador ......... & 40 & 4 & 0,10 & Sta. Lucía ........ & 37 & 7 & 0,19 \\
\hline Guatemala ....... & 45 & 3 & 0,07 & S. V. y Gra. ...... & 37 & 6 & 0,16 \\
\hline Honduras ........ & 47 & 4 & 0,09 & Trin. y Tob. ...... & 31 & 6 & 0,19 \\
\hline
\end{tabular}


Pasemos ahora a analizar ese envejecimiento a escala de país, en los ámbitos americano y europeo que son los que nos interesan en este momento (Cuadros I y II). Porcentaje de jóvenes y viejos e índice de envejecimiento de los países de América y Europa.

\section{Cuadro II}

PORCENTAJE DE JOVENES Y VIEJOS E ÍNDICE DE ENVEJECIMIENTO DE LOS PAISES EUROPEOS (AÑO 1995)

\begin{tabular}{|c|c|c|c|c|c|c|c|}
\hline Países & $\begin{array}{c}\% \\
\% \\
\text { menos } \\
\text { de } 15 \\
\text { años } \\
J\end{array}$ & $\begin{array}{c}\% \\
\text { más } \\
\text { de } 65 \\
\text { años } \\
V\end{array}$ & $\begin{array}{c}\text { Índice } \\
\text { de } \\
\text { envejeci- } \\
\text { miento } \\
\text { V/J }\end{array}$ & Países & $\begin{array}{c}\% \\
\text { menos } \\
\text { de } 15 \\
\text { años } \\
J\end{array}$ & $\begin{array}{c}\% \\
\text { más } \\
\text { de } 65 \\
\text { años } \\
V\end{array}$ & $\begin{array}{c}\text { Índice } \\
\text { de } \\
\text { envejeci- } \\
\text { miento } \\
\mathbf{V} / \mathbf{J}\end{array}$ \\
\hline Europa ...... & 20 & 13 & 0,65 & Liechtenstein ... & 19 & 10 & 0,53 \\
\hline Alemania ........ & 16 & 15 & 0,94 & Suiza ............. & 16 & 15 & 0,94 \\
\hline Bélgica .......... & 18 & 16 & 0,88 & Belorusia ........ & 22 & 12 & 0,55 \\
\hline Dinamarca ....... & 17 & 15 & 0,88 & Bulgaria .... & 19 & 14 & 0,74 \\
\hline España .......... & 17 & 15 & 0,88 & R. Checa ......... & 21 & 10 & 0,48 \\
\hline Francia .......... & 20 & 15 & 0,75 & Hungría .......... & 19 & 14 & 0,74 \\
\hline Grecia ........... & 29 & 14 & 0,74 & Moldavia .. & 28 & 9 & 0,32 \\
\hline Holanda .......... & 18 & 13 & 0,72 & Polonia ........... & 24 & 11 & 0,46 \\
\hline Irlanda $\ldots . . . . .$. & 26 & 11 & 0,42 & Rumanía ......... & 22 & 11 & 0,50 \\
\hline Italia .............. & 16 & 16 & 1,00 & Rusia .............. & 22 & 11 & 0,50 \\
\hline Luxemburgo .... & 18 & 14 & 0,80 & Eslovaquia . & 25 & 11 & 0,44 \\
\hline Portugal .......... & 18 & 14 & 0,80 & Verania ........... & 21 & 13 & 0,62 \\
\hline R. Unido ......... & 19 & 16 & 0,84 & Albania ........... & 33 & 5 & 0,15 \\
\hline Austria ........... & 18 & 15 & 0,83 & Bosnia ............ & 23 & 7 & 0,70 \\
\hline Estonia .......... & 21 & 13 & 0,62 & Croacia ........... & 19 & 13 & 0,68 \\
\hline Finlandia ......... & 19 & 14 & 0,72 & Macedonia ....... & 26 & 7 & 0,27 \\
\hline Islandia........ & 25 & 11 & 0,44 & Malta ............. & 23 & 11 & 0,48 \\
\hline Latvia ............ & 22 & 13 & 0,59 & S. Marino ... & 15 & 14 & 0,98 \\
\hline Lituania .......... & 22 & 12 & 0,55 & Eslovenia ........ & 19 & 12 & 0,63 \\
\hline Noruega .......... & 19 & 16 & 0,84 & Yugoslavia ....... & 23 & 10 & 0,43 \\
\hline Suecia ........... & 19 & 18 & 0,95 & & & & \\
\hline
\end{tabular}

Fuente: Data Sheet y elaboración propia.

Como puede extraerse de la observación de los cuadros anteriores, es evidente que el envejecimiento de los distintos países americanos y europeos está íntimamente relacionado no ya sólo con el gra- 
do o nivel de desarrollo, sino con la caída vertiginosa de la fecundidad. Los países americanos, aunque destaquen por su menor proporción de ancianos, lo hacen mucho más por su elevadísima proporción de jóvenes, lo que constituye sin duda la gran esperanza de su futuro. Se ha establecido, de forma un tanto ficticia, una clara relación biunívoca de ambos indicadores. A mayor nivel de desarrollo se le asigna o espera un mayor índice de envejecimiento. Si esto puede ser relativamente verdad a escala nacional, y como consecuencia de la propia historia demográfica, no puede, de ninguna manera, hacerse extensivo a otras escalas espaciales más reducidas.

\section{El envejecimiento demográfico en España}

Una vez realizada esta breve panorámica de la situación mundial, en la que se han podido comprobar los fuertes contrastes espaciales del envejecimiento demográfico a escala planetaria, continental, subcontinental y nacional, vamos a pasar a analizarlo en un espacio mucho más reducido, el de un solo país, España, y esencialmente en una parte de dicho espacio nacional, el mundo rural. Este espacio nacional, dividido según el criterio dicotómico rural/urbano, presenta contrastes que hemos de estudiar para poder extraer algunas causas y consecuencias a tener en consideración a la hora de elaborar y aplicar la política demográfica más acertada para la mejor calidad de vida de la población.

España, país en el que los efectos derivados de la Revolución Industrial se producen siempre con un cierto retraso, comparado con el resto de los países de Europa occidental, como consecuencia del propio retraso con el que se produjo dicha revolución, es también uno de los últimos países europeos en ver cómo se produce el envejecimiento de su población. Sin embargo, al igual que sucede con otros acontecimientos, la intensidad con la que tiene lugar es incluso mayor que en el resto de Europa occidental. Actualmente el índice de envejecimiento que presenta nuestro país sólo es superado por Italia, Suecia, Alemania y Suiza. Bélgica y Dinamarca poseen la misma cifra que España $(0,88)$. Pero el porcentaje de personas de más de 65 años es sólo más reducido que el de Suecia, Bélgica, Italia, Reino Unido y Noruega. ¿Quiere eso decir que el nivel de desarrollo español es se- 
mejante al de los citados países? Evidentemente no, simplemente se pone de manifiesto que en el proceso de transformación de la sociedad de agraria a industrial, que muestra de forma muy clara el modelo de transición demográfica, España se encuentra ya en la última fase del mismo, al igual que los restantes países de Europa occidental. El fuerte descenso de la fecundidad y de la mortalidad pueden llegar a producir una estructura de la población, por grupos de edad, como la que acabamos de ver.

\section{CuAdro III}

EVOLUCIÓN DE LA POBLACIÓN JOVEN Y VIEJA EN ESPAÑA EN EL SIGLO XX

\begin{tabular}{|c|c|c|c|}
\hline Año & $\begin{array}{c}\text { \% menores } \\
\text { de } 15 \text { años } \\
(J)\end{array}$ & $\begin{array}{c}\text { \% mayores } \\
\text { de } 65 \text { años } \\
\text { (V) }\end{array}$ & $\begin{array}{c}\text { Indice de } \\
\text { envejecimiento } \\
(\mathrm{V} / \mathrm{J})\end{array}$ \\
\hline 1900 & 33,5 & 5,2 & 0,16 \\
\hline 1910 & 33,9 & 5,5 & 0,16 \\
\hline ............... & 30,7 & 6,1 & 0,20 \\
\hline ...................... & 26,2 & 7,3 & 0,29 \\
\hline$\ldots \ldots \ldots \ldots \ldots \ldots \ldots$ & 27,9 & 9,8 & 0,35 \\
\hline 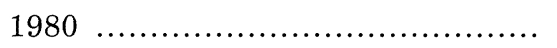 & 25,7 & 11,2 & 0,44 \\
\hline 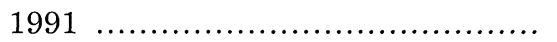 & 20,0 & 13,0 & 0,65 \\
\hline 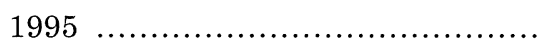 & 17,0 & 15,0 & 0,88 \\
\hline
\end{tabular}

FUENTE: INE.

Es curioso observar que, dentro de las fronteras de un determinado país, este indicador de envejecimiento, que responde a una idea general de que a mayor envejecimiento mayor grado de desarrollo, se convierte, en esa escala intranacional, en algo totalmente opuesto.

Si bien es verdad que todos los autores coinciden en citar como cuantitativamente más numeroso el envejecimiento del mundo urbano y, fundamentalmente, por el cambio que supone, ya que la ciudad se ha caracterizado siempre por la mayor proporción de jóvenes; el mundo rural, ya tradicionalmente más envejecido, es actualmente el que presenta un envejecimiento mayor en el sentido estructural. Con

$$
-578-
$$


la nueva configuración espacial adquirida por las ciudades (aparición y desarrollo de las áreas metropolitanas, en las que el precio mayor del suelo, en el centro de la ciudad tradicional, expulsa a los jóvenes a municipios metropolitanos, donde, al precio más barato del suelo que se refleja en un menor precio de la vivienda hay que añadir la atracción que supone la aparición de nuevos modelos de urbanización realizados en estos últimos, y que llevan consigo un gran cambio en los modos de vida, resultando más atractivos para personas de menor edad, entre otras razones), el espacio ocupado por la ciudad tradicional aparece hoy muy envejecido, sobre todo en contraste con su área metropolitana. Dicho espacio central urbano sufre hoy una serie de problemas, consecuencia no siempre del envejecimiento de sus habitantes, que, en cierto modo, se asemejan a los del mundo rural.

La distribución del envejecimiento, a escala municipal en el total nacional, muestra cómo los municipios con porcentajes de personas mayores superiores al 30 \% son más numerosos en la España septentrional que en la meridional. Algo similar se observa entre la occidental y la oriental. La mayor concentración de este tipo de municipios se encuentra en el área comprendida entre el sistema Ibérico y las estribaciones orientales del Sistema Central, y en general manifiestan una desigualdad espacial entre un norte más envejecido y el sur relativamente más joven (Paniagua, A., y López, J. J., 1991). Estos autores señalan como causa de ello el carácter emigratorio de estas áreas hacia las más industrializadas, frente a la fecundidad más elevada tradicional de las provincias del sur que compensó la emigración de estas regiones españolas. Es decir, las provincias andaluzas, a pesar de ser de fuerte tendencia emigratoria, siguen siendo jóvenes gracias a su tradicional elevada fecundidad. Se cumple pues, lo que señalaba Myers en 1985 y que recogen los mismos autores en otro de sus trabajos: «la transición demográfica demuestra que al dejar de crecer una población, envejece, de forma que el mejor antídoto contra el envejecimiento es el crecimiento».

Las causas del mayor envejecimiento del mundo rural español se pueden resumir en las siguientes:

1) Históricamente el responsable fue el éxodo rural. La emigración hacia las zonas urbanas supuso una gran sangría de población para el campo, fundamentalmente en las décadas posteriores a la 
guerra civil, que originó el descenso de la población rural en edades fértiles. Ello redujo la natalidad, y, dadas las mejoras sanitarias conseguidas, una disminución de la tasa de mortalidad. Ambos hechos llevan a una sociedad al envejecimiento, puesto que cada vez habrá mayor proporción de personas de más de 65 años y menor proporción de las menores de 15 años.

2) En la actualidad, al de por sí ya elevado envejecimiento estructural de la población residente se suma el hecho de la aparición, de unos años a esta parte, de un cierto movimiento de retorno hacia sus pueblos de aquellos emigrantes que se fueron a trabajar en la industria en los años sesenta. Lógicamente, son jubilados, la mayoría de ellos con edades avanzadas, pero que el escaso valor adquisitivo de su pensión, la falta o deterioro de su vivienda en la ciudad, la vida menos cómoda o más agresiva para las personas mayores de nuestras ciudades, la existencia de residencias de pensionistas, etc., producen este movimiento de personas desde la ciudad hacia el campo y fundamentalmente de personas de más de 65 años.

\section{El envejecimiento del mundo rural aragonés}

Una vez contemplada la distribución del envejecimiento en el total del espacio nacional español, vamos a centrarnos en el análisis de detalle de una de sus regiones autonómicas: Aragón Hemos elegido este espacio peninsular porque se trata de la Comunidad Autónoma con mayor proporción de personas de más de 65 años en 1986 (15,54\%), más de tres puntos por encima de la media nacional $(12,18)$ en ese año. Corresponde dicho espacio peninsular al arco mediterráneo, con una economía diversificada, ya que a la tradicional economía agraria se suma la industrial y la de los servicios, aunque muy concentrada en las capitales provinciales y algunos municipios de mayor desarrollo tradicional.

Aragón, una de las 17 regiones autonómicas españolas, se extiende sobre una superficie de $47.669 \mathrm{~km}^{2}$, que suponen el $9,5 \%$ de la superficie nacional y la habitan el 3,06 \% de los españoles. Esta región humana está formada por tres unidades naturales que los acontecimientos históricos han integrado: Los Pirineos, La Depresión del Ebro y El Sistema Ibérico. La primera y última unidad son dos siste- 
mas montañosos unidos por la tierra llana central regada por el río Ebro. En cuanto a la proporción de municipios rurales que componen esta autonomía y el total nacional son las siguientes: de los $728 \mathrm{mu}$ nicipios aragoneses existentes en 1991, 678 tienen menos de 2.000 habitantes, lo que significa el $93 \%$ del total. España está formada por 8.077 municipios en ese mismo año, de ellos 5.944 son rurales, lo que supone el $73 \%$ del total. En cuanto a la población que habita estos municipios son 3.115.007 personas en España y 259.422 en Aragón, ello supone el $8 \%$ y el 21,8 \% respectivamente. Estos datos permiten hacernos una idea aproximada de las semejanzas y diferencias existentes entre este espacio autonómico y el nacional en cuanto a ruralidad y población se refiere.

Estudiando en su totalidad este espacio, presenta una proporción de personas de más de 65 años del 19,71\% en 1991. Huesca, Teruel y Zaragoza, las tres provincias que componen esta región son de naturaleza física, humana y económica muy diferente. En ellas, consideradas en su totalidad, la proporción de personas de más de 65 años es de $20,47 \%, 22,20 \%$ y $16,45 \%$ respectivamente. Pero estas cifras resultan del promedio de dos ámbitos muy diferentes el rural y el urbano.

Esta Comunidad Autónoma, la aragonesa, está compuesta por 728 municipios. De los cuales sólo 55 tienen más de 2.000 habitantes, es decir, tan sólo el 7,6\% de ese espacio está organizado por núcleos de población que pueden considerarse semiurbanos o urbanos, aplicando la escala señalada por el INE español. Pero aún podemos deslindar más las diferencias entre los espacios provinciales. Mientras la provincia de Zaragoza cuenta con 29 (9,9\%) de estos municipios no rurales, Huesca posee tan sólo $12(6,0 \%)$ y en la provincia de Teruel hay todavía un número menor, 10 (4,2\%) municipios con más de esa población. Está claro, pues, que si bien se trata de una región que, como toda España, está organizada por las áreas urbanas, en este caso fundamentalmente por la macrocefálica región urbana de Zaragoza, que supone el 71 \% de la población aragonesa, son los escasos habitantes del enorme espacio ocupado por los municipios rurales los responsables de la explotación, generalmente agraria, la obtención de los máximos rendimientos del campo, la ordenación y cuidado de ese gran espacio, en el que la problemática economía actual hace responsable de su desarrollo. 
Veamos ahora cuál es la diferenciación espacial del envejecimiento en ese gran espacio rural. En el cuadro núm. III se expresa la proporción de personas de más de 65 años en el total de los municipios de esta Comunidad Autónoma. En el núm. IV se inscribe la misma proporción pero sólo en los municipios rurales.

\section{Cuadro IV}

DISTRIBUCIÓN DE LOS MUNICIPIOS ARAGONESES SEGÚN LA PROPORCIÓN DE PERSONAS DE MAS DE 65 AÑOS

\begin{tabular}{|c|c|c|c|c|c|c|c|c|c|c|c|}
\hline$\%+65$ años & & $15 \%$ & & $-20 \%$ & & $-25 \%$ & & $-30 \%$ & & $-30 \%$ & $\begin{array}{l}\mathrm{N}^{\mathrm{o}} \\
\text { total }\end{array}$ \\
\hline Huesca & 4 & $2,0 \%$ & 26 & $12,9 \%$ & 44 & $21,8 \%$ & 66 & $32,7 \%$ & 62 & $30,7 \%$ & 202 \\
\hline Teruel ........ & 3 & $1,3 \%$ & 12 & $5,1 \%$ & 39 & $16,5 \%$ & 65 & $27,5 \%$ & 117 & $49,6 \%$ & 234 \\
\hline Zaragoza ..... & 12 & $4,2 \%$ & 31 & $10,6 \%$ & 51 & $17,5 \%$ & 88 & $30,2 \%$ & 109 & $37,4 \%$ & 292 \\
\hline Aragón ....... & 19 & $2,7 \%$ & 69 & $9,3 \%$ & 134 & $18,2 \%$ & 219 & $29,7 \%$ & 238 & $39,0 \%$ & 738 \\
\hline
\end{tabular}

\section{Cuadro V}

DISTRIBUCIÓN DE LOS MUNICIPIOS RURALES ARAGONESES SEGÚN LA PROPORCIÓN DE PERSONAS DE MAS DE 65 AÑOS

\begin{tabular}{|c|c|c|c|c|c|c|}
\hline$\%+65$ años & $-15 \%$ & $15-20 \%$ & $20-25 \%$ & $25-30 \%$ & $+30 \%$ & $\mathrm{N.}_{\text {total }}^{\mathrm{o}}$ \\
\hline Huesca & $31,5 \%$ & $188,9 \%$ & $42 \quad 20,8 \%$ & $65 \quad 32,2 \%$ & $6230,7 \%$ & 190 \\
\hline Teruel .......... & $10,4 \%$ & $73,0 \%$ & $36 \quad 15,3 \%$ & $65 \quad 27,1 \%$ & $11749,6 \%$ & 225 \\
\hline Zaragoza ........ & $10,4 \%$ & $186,2 \%$ & $42 \quad 14,4 \%$ & $8829,8 \%$ & $10937,4 \%$ & 263 \\
\hline Aragón ......... & $111,6 \%$ & $43 \quad 6,3 \%$ & $120 \quad 17,7 \%$ & $21831,9 \%$ & $28842,5 \%$ & 678 \\
\hline
\end{tabular}

En ambos cuadros se han clasificado los municipios según el criterio establecido por Paillat y Parant (1983) para los municipios rurales franceses, aunque adaptado a la realidad del mundo rural aragonés. Estos autores consideran municipios rurales jóvenes aquellos cuya proporción de ancianos es inferior al $15 \%$, municipios en transi-

$$
-582-
$$


ción los que tienen entre el 16 y el $19 \%$, viejos los que poseen valores entre el 20 y el $23 \%$, y muy viejos si tienen un $24 \%$ o más. Nosotros hemos añadido nuevos intervalos, dejando como muy viejos los municipios con porcentajes comprendidos entre el 25 y el $30 \%$ y muy muy viejos los que superan el $30 \%$. Los porcentajes obtenidas en ambos cuadros nos confirman el alto grado de envejecimiento de esta región española en general, pero particularmente de su extenso, pero escasamente poblado, mundo rural. Sólo el $7,3 \%$ de los municipios rurales aragoneses se podrían clasificar como jóvenes o en transición en la terminología de Paillat, o, lo que parece aún más llamativo, el 92,7 \% son viejos, muy viejos o muy muy viejos.

Por provincias, es Teruel la más envejecida y la que presenta mayor proporción de municipios en estadios envejecidos. Es la provincia en la que se reúnen dos importantes factores negativos para el crecimiento de la población. A un medio físico hostil (altitud, clima, suelos...) se suma su lejanía de centros urbanos dinámicos. Las vías, tanto de comunicación como fluviales, son también escasas, con lo que tampoco pueden posibilitar el desarrollo de actividades que le hagan atraer a la población. Sólo la capital provincial y Alcañiz pueden considerarse urbanos, al tener más de 10.000 habitantes. Los restantes municipios, los considerados como semiurbanos, son antiguas cabeceras de comarca; Andorra y Calamocha se encuentran en el intervalo de 2.000 a 10.000 habitantes, pero su dinamismo se reduce al de regir una más amplia zona rural, con una economía poco diversificada y más centrada en la oferta de servicios comarcales.

La provincia de Huesca presenta un porcentaje similar de personas de más de 65 años que la de Teruel, ambas superan el $20 \%$, aunque la que nos ocupa posee tan sólo el $20,47 \%$ frente al 22,20\% de la segunda. Es también una provincia con un medio físico hostil, sobre todo por su altitud, pero sin embargo en ella se encuentra el máximo número de núcleos de entre las tres provincias. Huesca, Monzón, Barbastro, Jaca y Fraga superan los 10.000 habitantes y tienen algunas de ellas una economía más diversificada, tradición industrial y de servicios que las hacen atractivas para la población. Por lo demás, sólo siete municipios tienen entre 2.000 y 10.000 h., es decir, son semiurbanos. El dinamismo demográfico provincial es reducido, y sólo el $10,4 \%$ de los municipios rurales pueden considerarse jóvenes o en 
transición. El 89,6 \% restante se distribuye entre los viejos, muy viejos o muy muy viejos, como puede observarse en el cuadro.

La provincia de Zaragoza es la más joven de las tres, el 16,45\% de su población tiene más de 65 años. En esta provincia coinciden factores positivos al establecimiento de la población. El valle del Ebro, con una menor altitud, las vías de comunicación que corren paralelas al río, y las que comunican con los cuatro vértices de un cuadrado formado por Bilbao, Barcelona, Valencia y Madrid, y que confluyen o se cruzan en la capital provincial. Todo ello propicia el desarrollo, junto al de la agricultura de regadío tradicional, el de áreas industriales y de servicios y de servicios capaces de ofrecer mayores posibilidades a la población.

Zaragoza posee cuatro núcleos urbanos. Junto a la capital provincial, con más de 500.000 h., otros tres municipios: Calatayud, Ejea de los Caballeros y Tarazona superan los 10.000 habitantes. Però además otros 25 tienen más de 2.000 h. No obstante, a pasar de tener una red urbana mejor configurada, no funciona como auténtica red, ya que fallan las conexiones y Zaragoza ha sido tradicionalmente la que ha succionado a la población aragonesa, creciendo ella y dejando tras sí un gran desierto hasta la siguiente ciudad en tamaño que es Huesca. Actualmente Zaragoza concentra a casi las tres cuartas partes de la población aragonesa, y si exceptuamos las comarcas oscenses, más dinámicas económicamente, Zaragoza concentra también la mayor parte de los recursos económicos, ya que en ella se generan prácticamente todas las rentas aragonesas procedentes de la industria y los servicios.

En la actualidad, se detecta, como en otras reglones urbanas españolas, que Zaragoza ha generado un efecto metropolitano que se extiende de momento a su entorno más próximo. Este efecto es el rejuvenecimiento, o mejor, el aumento de la fecundidad en algunos de los municipios que la rodean. Muchos de ellos, están todavía hoy poco poblados, pero sin embargo, presentan una proporción de personas mayores de 65 años no muy elevada. Los matrimonios jóvenes que se instalan en ellos producen un efecto positivo sobre la natalidad.

En la figura 1 se ha representado la distribución espacial del envejecimiento de los municipios rurales aragoneses. En ella se aprecian con claridad muchos de los aspectos espaciales que señalábamos anteriormente. En primer lugar la concentración de la población en el 

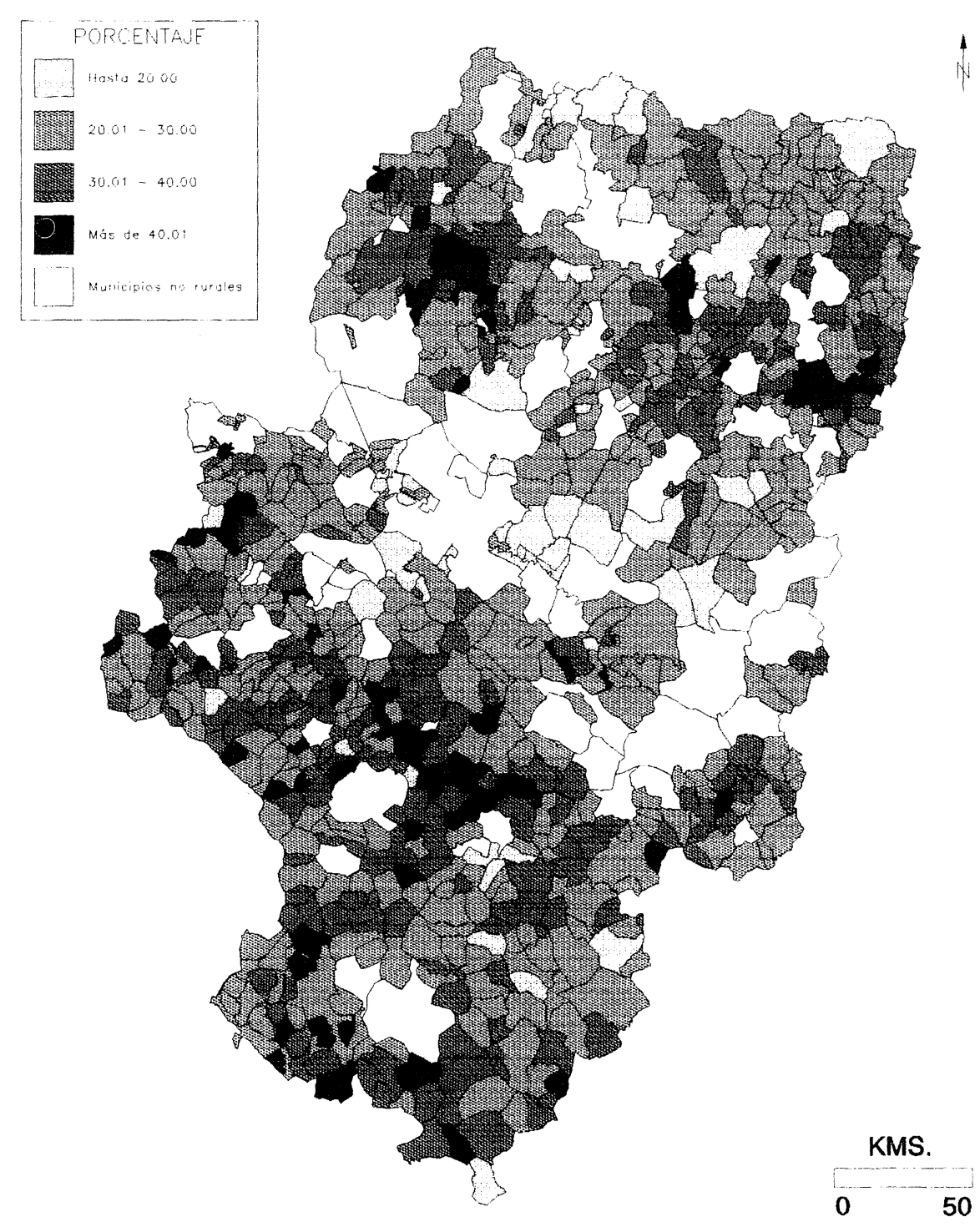

Figura 1.-Aragón \% de mayores de 65 años en municipios rurales 
Valle del Ebro, municipios industrializados del norte de Huesca y capital provincial, y cabeceras comarcales en la de Teruel. Son los municipios que aparecen en blanco en el mapa, identificados como no rurales. En segundo lugar, el gran número de municipios rurales que componen esta región. En estos se ha representado la proporción de mayores de 65 años según la escala de grises que figura en el mapa. Se observa que los tonos más oscuros se concentran en los municipios más elevados y menos accesibles del Pirineo oscense y del Sistema Ibérico zaragozano y turolense, aunque con valores más elevados y mayor número de municipios en este último. Una vez más se pone de manifiesto la influencia de un medio fisica accidentado como factor negativo a la instalación de la población. La correlación clara entre mayor altitud-menor población se evidencia aquí de forma que a mayor altitud le corresponde también mayor envejecimiento, indudablemente debida a la escasa población, como veremos después.

Los municipios más envejecidos, con más de un $40 \%$ de ancianos, son muy numerosos. En la provincia de Huesca el más envejecido es Baélls con el $45,6 \%$ de sus 171 habitantes superando esa edad. Agüero (168 h.), Bierge (198 h.), Castillazuelo (252 h.), Estopiñán del Castillo (184 h.), Palo (33 h ), Peralta de Calasanz (349 h.) y Quicena (160 h.) están próximos a esa cifra superando el $40 \%$. Por último, destacar que el $62,7 \%$ de los municipios rurales oscenses tienen una población de más de 65 años que supera el $25 \%$, es decir, son muy viejos. La provincia de Teruel es la más envejecida, la que posee municipios más envejecidos y mayor número de ellos El municipio de Salcedillo, con 9 habitantes, posee el máximo porcentaje de ancianos, el $100 \%$, es decir, todos sus habitantes tienen más de 65 años. A Sacedillo le siguen Maicas, en el que, de los $20 \mathrm{~h}$. que lo componen, un $65 \%$ tiene más de esa edad, y El Vallecillo, en el que el 60,0\% de sus 25 habitantes son ancianos. Pero esta provincia todavía tiene 6 municipios con más de la mitad de su población anciana: Allueva, Plou, Josa, Alcaine, Cubla y Alobras, con población que oscila entre los 12 y los 120 habitantes. Los que superan el $40 \%$ de viejos son todavía 21 municipios, la mayor parte de ellos con menos de $100 \mathrm{~h}$. y el resto están entre 100 y 200 h. a excepción de Loscos que tiene 210 h. Por último, señalar que en esta provincia, la proporción de municipios rurales muy viejos supera el $76 \%$. 
La provincia de Zaragoza, gracias al mayor número de núcleos urbanos, y al dinamismo de su capital, es la menos envejecida. Sin embargo, contiene municipios rurales muy envejecidos y un elevado número de ellos, como se puede ver en el mapa. Pomer, municipio de 25 h., es el más envejecido, con un $80 \%$ de su población con más de 65 años. Le sigue Cerveruela, que con $4 \mathrm{~h}$. es el municipio menos poblado de toda España, y el $75 \%$, es decir, 3, superan esa edad. A éste le sigue Balconchán, en el que el $70,51 \%$ de sus 34 habitantes es mayor de 65 años. Por encima del $50 \%$ de ancianos están 8 municipios: Oseja, con 30 h. y el 56,7 \%, Moneva, con 110 h. y 56,4 \%, Grisel, con 58 h. y el 55,935\%, Almochuel, con 53 h. y 54,7\%, Fombuena, con 13 h. y el 53,85\%, Undués de Lerda con 47 h. y el 53,2\%, Calcena con $105 \mathrm{~h}$ y $52,4 \%$ y Talamantes, con $64 \mathrm{~h}$. y el $51,6 \%$ de ellos mayores de 65 años. A estos 11 municipios hay que sumarles otros 17 que superan el $40 \%$ de ancianos, la mayoría de ellos con menos de 100 habitantes y todos, a excepción de Sta. Cruz de Grío que tiene $238 \mathrm{~h}$, con menos de $200 \mathrm{~h}$.

Esta distribución espacial del envejecimiento de los municipios rurales aragoneses pone de manifiesto la íntima relación del envejecimiento con el pequeño volumen de población municipal. De lo analizado hasta aquí se puede afirmar que los municipios más envejecidos tienen poblaciones por debajo de los $200 \mathrm{~h}$, y una gran mayoría, por debajo de 100. Se trata de municipios de economía agraria dominante, con una agricultura o ganadería extensivas, ocupando tierras marginales, cuya población obtiene rendimientos muy bajos de su trabajo. La emigración hacia Cataluña o América en los años 50, y hacia Zaragoza o Europa en los 60, dejó este territorio sin los grupos de edad más fértiles, lo que produjo un gran descenso de la fecundidad.

Este círculo vicioso en el que se inscribe el mundo rural aragonés, en el que al tradicional flujo migratorio se suma la baja fecundidad, produce un envejecimiento que provoca la falta de dinamismo económico de su sector agrario, a excepción de las áreas de regadlo. A su vez, se van desertizando grandes extensiones, algunas ya tradicionales, como Los Monegros, y los Somontanos Ibérico y Pirenaico

Desde 1960, el número de municipios se ha reducido en España en casi 2.000, como puede observarse en el cuadro núm. VI. El $10 \%$ de ellos han desaparecido en Aragón, y ésta es la tendencia que se 
puede prever para el futuro, según lo que acabarnos de analizar. De las tres provincias, es Huesca la que ha visto desaparecer un mayor número de ellos, 147 en 31 años, 48 ha perdido la de Teruel, y sólo 12 la de Zaragoza.

Cuadro VI

EVOLUCIÓN DEL NÚMERO DE MUNICIPIOS

\begin{tabular}{|c|c|c|c|}
\hline & 1960 & 1970 & 1991 \\
\hline Huesca & 349 & 254 & 202 \\
\hline Teruel $\ldots . . . . . . . .$. & 282 & 268 & 234 \\
\hline Zaragoza & 304 & 298 & 292 \\
\hline Aragón & 935 & 820 & 728 \\
\hline España & 9.902 & 8.655 & 8.077 \\
\hline
\end{tabular}

\section{Cuadro VII}

\section{MUNICIPIOS OSCENSES CON ÍNDICE DE ENVEJECIMIENTO POR DEBAJO DE LA UNIDAD}

\begin{tabular}{|c|c|c|c|c|c|}
\hline Municipio & Población & $\begin{array}{l}\text { Índice de } \\
\text { envejeci- } \\
\text { miento }\end{array}$ & Municipio & Población & $\begin{array}{l}\text { Índice de } \\
\text { envejeci- } \\
\text { miento }\end{array}$ \\
\hline Banastas & 110 & 0,65 & Monzón & 14.690 & 0,95 \\
\hline Barbastro ............ & 14.778 & 0,94 & Panticosa ............ & 689 & 0,89 \\
\hline Benasque $\ldots . . . \ldots \ldots$ & 1.081 & 0,69 & Sabiñánigo .......... & 9.056 & 0,77 \\
\hline Fraga ................ & 11.491 & 0,89 & Sallent .............. & 886 & 0,87 \\
\hline Huesca ......... & 44.165 & 0,88 & Vencillón & 514 & 0,95 \\
\hline Jaca ................ & 10.840 & 0,99 & & & \\
\hline
\end{tabular}

Si además del envejecimiento, estudiado como la proporción de personas de más de 65 años que componen la población municipal, analizamos el índice de envejecimiento, poniendo en relación el número de viejos y jóvenes $(\mathrm{V} / \mathrm{J})$, la tendencia al envejecimiento e in-

$$
-588-
$$


cluso a la desaparición de los municipios rurales aragoneses es mucho más evidente.

Los índices de envejecimiento provinciales son del 2,20 en Teruel, del 1,30 en Huesca y del 1,0 en Zaragoza. Ello viene a confirmar, e incluso a reforzar, la idea del envejecimiento de la población aragonesa que expresaba el porcentaje de mayores de 65 años. Estos índices provinciales, de por si muy elevados con respecto a la media nacional, 0,88 , se ven muy incrementados al estudiar los municipios rurales. Muchos de ellos no tienen ningún niño menor de 15 años. Fanlo en la provincia de Huesca, Salcedillo en la de Teruel y Cerveruela, Fombuena, Godojos, Pomer, Pozuel de Ariza, Puendeluna, Purujosa y La Zaida, en la de Zaragoza. La existencia de 1 ó 2 niños menores de 15 años nos dan cifras tan extrañas como la de 55 en Velilla de Jiloca, de 33 en Talamantes, de 31 en Moneva, de 29,5 en Orés, de 27,5 en Calcena, de 27 en Los Pintanos, de 25 en Torrelapaja y de 21 en Las Caerlas, todos ellos en la provincia de Zaragoza. Entre valores del $10 \mathrm{y}$ el 20 se encuentran los índices de 6 municipios rurales oscenses, 8 de entre los turolenses y 12 de los zaragozanos, muchos de ellos coincidentes con los que tenían mayor proporción de ancianos. Valores muy elevados, entre el 2 y 10 los tienen la mayor parte de los restantes municipios rurales, y la proporción de los que presentan valores entre 1 y 2 es mucho más reducida. Sin embargo, por debajo del 1 son escasísimos, por lo que será más significativo su análisis.

En la provincia de Huesca sólo 11 municipios tienen un índice de envejecimiento por debajo de la unidad. De ellos, tan sólo cinco son rurales, es decir el 5,4\% de total municipal provincial y el $2,4 \%$ de los municipios rurales. En el cuadro núm. VII se recogen los datos de estos municipios Pero, ¿qué tipo de economía dirige esa población? Como puede observarse en el cuadro, se trata de 6 municipios rurales y 5 urbanos. Estos últimos, junto a la capital provincial, son los municipios de tradición industrial oscense. Todos tienen un índice de envejecimiento superior al de los rurales, debido a que, seguramente, el volumen mayor de población lo hace más estable Los rurales, son municipios con un gran desarrollo del sector terciario, gracias a las ofertas turísticas de algunos valles pirenaicos, tanto para deportes de invierno (Benasque, Sallent de Gállego), como de balnearios (Panticosa), se beneficien del dinamismo económico, no ya sólo de la capital regional, Zaragoza, sino de las limítrofes, como las del País Vasco, 
La Rioja, Cataluña, e incluso de la de Madrid. Ello le confiere un interés económico extrarregional.

En la provincia de Teruel hay, por el contrario, mayor número de municipios con índice de envejecimiento inferior a la unidad, 33, y los valores que alcanzan son incluso menores que los que acabamos de ver en la de Huesca En el caso de la provincia turolense, cuyos datos se pueden observar en el cuadro núm. VIII, la explicación está menos clara. A excepción de los municipios no rurales, Utrillas y Andorra, no se puede hablar de dinamismo demográfico. Estos municipios menos envejecidos se localizan, fundamentalmente, en las Sierras de Cucalón y de Gúdar, próximos al valle del Alfambra. Aquí, a la actividad agraria dominante, se puede añadir un cierto efecto beneficioso del turismo. Los restantes municipios, distribuidos por el resto de la provincia, tienen como denominador común el estar próximos a las redes de comunicación, aspecto del que también gozaba el grupo anterior, y en el área de influencia de otros municipios dinámicos. Calaceite, en la depresión del Ebro, tiene ya suficiente entidad como para pensar un dinamismo propio Pero, seguramente, dado el escaso volumen de población, una estructura por edades más equilibrada en la base y vértice de la pirámide, nos darían los índices de envejecimiento que se obtienen.

La provincia de Zaragoza posee 17 municipios con un índice de envejecimiento inferior a la unidad. En el cuadro núm. IX se pueden observar estos datos. Aquí, tanto los municipios rurales como muchos de los no rurales, se ven netamente respaldados por los benéficos influjos del dinamismo de la macrocefálica región urbana zaragozana. El valle del Ebro, de baja altitud, con una rica huerta, con unas facilidades de comunicación considerables, son factores favorecedores de ese dinamismo que se unen a los que supone la proximidad urbana.

En el caso zaragozano hay también algunos casos que, a causa de su menor población, pueden tener una explicación similar a la que veíamos en la provincia de Teruel, pero aquí y en la mayoría de los casos, se debe a la gran influencia de la capital. Una gran parte de ellos está a menos de $30 \mathrm{kms}$ de Zaragoza, y esta influencia se detecta tanto en los municipios no rurales (Utebo, es un no rural a menos de $5 \mathrm{kms}$ ) como en rurales (Figueruelas, municipio rural en el que se instaló la factoría de la General Motors). 


\section{CuAdro VIII}

\section{MUNICIPIOS TUROLENSES CON ÍNDICE DE ENVEJECIMIENTO} POR DEBAJO DE LA UNIDAD

\begin{tabular}{|c|c|c|c|c|c|}
\hline Municipio & Población & $\begin{array}{l}\text { Índice de } \\
\text { envejeci- } \\
\text { miento }\end{array}$ & Municipio & Población & $\begin{array}{c}\text { Índice de } \\
\text { envejeci- } \\
\text { miento }\end{array}$ \\
\hline Aguatón ................ & 32 & 0,48 & Maicas .. & 20 & 0,72 \\
\hline Alba .......... & 328 & 0,66 & Martín del Río & 574 & 0,82 \\
\hline Alcalá de la Selva ... & 462 & 0,12 & Miravete de la S. .... & 49 & 0,62 \\
\hline Allepuz ............... & 147 & 0,52 & Monforte de Moyu. .. & 116 & 0,27 \\
\hline Anadón ................. & 28 & 0,02 & Monroyo ............... & 116 & 0,64 \\
\hline Andorra & 8.680 & 0,68 & Nogueras & 31 & 0,92 \\
\hline Bea ................... & 28 & 0,26 & Obón $\ldots . . . . . . . . . . . . . .$. & 70 & 0,96 \\
\hline Cabra de Mora ...... & 102 & 0,26 & Palomar de Arroyos & 320 & 0,96 \\
\hline Calaceite ............. & 1.281 & 0,97 & Pozuelo del Campo .. & 148 & 0,83 \\
\hline Camarillas ............. & 132 & 0,73 & Saldón .................. & 53 & 0,50 \\
\hline Castelnou ............. & 125 & 0,53 & Sta. Cruz de Nogu. .. & 32 & 0,22 \\
\hline El Castellar ........... & 103 & 0,45 & Terriente .............. & 205 & 0,09 \\
\hline Celadas ................ & 488 & 0,47 & Torrijas ......... & 74 & 0,94 \\
\hline Escorihuela ......... & 273 & 0,60 & Tronchón ............ & 119 & 0,78 \\
\hline Escucha .............. & 1.212 & 0,50 & Urrea de Gaen ....... & 700 & 0,50 \\
\hline Fuentes Calientes ... & 165 & 0,95 & Utrillas ............... & 3.748 & 0,79 \\
\hline Gudar .................. & 89 & 0,16 & Veguillas ............... & 33 & 0,80 \\
\hline
\end{tabular}

\section{Cuadro IX}

MUNICIPIOS ZARAGOZANOS CON ÍNDICE DE ENVEJECIMIENTO POR DEBAJO DE LA UNIDAD

\begin{tabular}{|c|c|c|c|c|c|}
\hline Municipio & Población & $\begin{array}{c}\text { Índice de } \\
\text { envejeci- } \\
\text { miento }\end{array}$ & Municipio & Población & $\begin{array}{c}\text { Índice de } \\
\text { envejeci- } \\
\text { miento }\end{array}$ \\
\hline Alfajarín ............. & 1.458 & 0,90 & Nuévalos ............. & 287 & 0,88 \\
\hline Alfamen .............. & 1.299 & 0,95 & Osera de Ebro ....... & 346 & 0,95 \\
\hline Anento ................ & 104 & 0,40 & Pinseque .............. & 1.363 & 0,95 \\
\hline Cadrete ............... & 917 & 0,67 & Puebla de Alfinden ... & 1.463 & 0,77 \\
\hline Cuarte de Huerva ... & 1.353 & 0,59 & Utebo .................. & 7.671 & 0,41 \\
\hline Ejea de los Cab. ..... & 15.337 & 0,75 & Villanueva de G. .... & 2.489 & 0,96 \\
\hline Figueruelas ........... & 870 & 0,79 & Zaida (La) ............ & 643 & 0,98 \\
\hline Fuentes de Ebro .... & 3.801 & 0,88 & Zaragoza .............. & 594.394 & 0,84 \\
\hline La Muela ............. & 1.006 & 0,92 & & & \\
\hline
\end{tabular}




\section{Conclusión}

Quizás sea mejor, para el bienestar de la población, la nueva distribución espacial que está generando la dinámica demográfica aragonesa. Tal como hemos visto, han desaparecido un gran número de municipios, y muchos otros lo harán en un futuro próximo. Seguramente, al igual que una sociedad, basada en la economía agraria, necesitaba de una distribución espacial de sus entidades de población muy semejante a la que descubrió Christaller que existía en el sur de su Alemania natal, y que cubría las necesidades de bienes y servicios de la población, debe existir, actualmente, un nuevo modelo de distribución, generado tras la revolución industrial, y con ella de la urbana, y que, como consecuencia de la revolución de los transportes establezca las directrices de su distribución. Pero el campo, transformado por el abandono, provoca problemas que se deben contemplar y tratar de solucionar. El crecimiento de ciudades intermedias, que organizaran los espacios intermedios despoblados, atendiendo las directrices de la Unión Europea, realizando plantaciones arbóreas en las tierras abandonadas, y procurando su conservación así como la mejora de las existentes, parece, a primera vista, la solución más idónea a los problemas planteados en estas extensas tierras de Aragón.

La forma en la que ha de llevarse a cabo es un reto para las distintas administraciones políticas, autonómica, nacional e internacional. De hecho, actualmente, se está viviendo una profunda transformación en el desarrollo de algunas de las actividades economices históricas y las consecuencias de otras acaecidas con anterioridad. Los bosques españoles están sufriendo incendios debidos, la mayoría de las veces, a causas no naturales, es decir, consecuencia de la acción humana, ya sea voluntaria o involuntaria. Las acciones voluntarias tienen su origen en la actuación de pirómanos, pagados por empresas que pretenden el abaratamiento de la madera, ya sea para pasta de papel o ya sea para la fabricación de muebles, o por empresas inmobiliarias que pretenden convertir en suelo urbanizable lo que hasta ese momento era terreno rústico. También las venganzas personales pueden ocasionar problemas, como sucedió en la isla de La Palma, hecho que no es único, y que se reproduce en otras autonomías. El problema de los incendios forestales, si bien no tiene una causa directa en la despoblación del mundo rural, sí puede ser indi-

$$
-592-
$$


rectamente una consecuencia de ello, sobre todo, si no de su origen, sí de su expansión. Si el bosque se mantuviera en mejores condiciones, nos referimos a que hasta hace relativamente poco tiempo los habitantes del mundo rural lo mantenían limpio de plantas favorables a la incineración, como ramas secas, aliagas, plantas que forman el sotobosque, etc., y se utilizaba para la leña necesaria en sus hogares o para el ganado, hoy en día esta tradicional labor ha desaparecido. La consecuencia inmediata es la necesidad de limpieza de ese sotobosque, realizada por personas que ya no van a constituir población activa agraria, sino del sector servicios, ya que los contratará la agencia del medio ambiente correspondiente en su autonomía. Estas agencias del medio ambiente serán las que retomen esa labor esencial para el buen funcionamiento del anterior ciclo biológico-humanoeconómico que permita la existencia de las mejores condiciones para la vida humana.

\section{BIBLIOGRAFIA}

Abellán García, A. (1990): El envejecimiento de la población, tendencias e implicaciones, IEGA, Departamento de Geografía Humana y Regional, CSIC, Documento de Trabajo núm. 1.

Abellán Garcia, A.; Fernandez-Mayoralas Fernandez, G.; Rodriguez Rodríguez, V.; Rojo Pérez, F. (1990): «El envejecimiento de la población española y sus características sociosanitariass», Estudios Geográficos Madrid, pp. 241-257.

Bodega Fernández, M. I.; García Alvarado, J. M.; Gutiérrez Ronco, S.; Martín Lou, M. A.; Muñoz Muñoz, J.; Navarro Madrid, A.; Casas Torres, J. M. (1986): «Densidades, población absoluta, y grados de envejecimiento de la población en la provincia de Guadalajara (España) en 1981», Geographica, Madrid, pp. 61-111.

CAmarero Rioja, L. A. (1992): «El mundo rural español en la década de los noventa: ¿renacimiento o reconversión?», Documentación social, Madrid, pp. 9-28.

Garcia Álvarez Coque, J. M.; Arnalte Alegre, E. (1990): «Población activa agraria durante el período de crisis», Agricultura y Sociedad, Madrid, pp. 117-154.

Garcia Ballesteros, A.; Brandis, D.; Troitiño, M. A. (1977): «Diferencias espaciales en el envejecimiento de la población rural española», $V$ Coloquio de Geografía, Granada, AGE, pp. 523-537.

LÓPEZ JIMÉNEZ, J. J. (1990): «Envejecimiento, tamaño demográfico y sector de actividad en los municipios españoles», Estudios Territoriales, Madrid, pp. ??

LÓPEZ JIMÉNEZ, J. J. (1992): «El proceso de envejecimiento demográfico en España», $R e$ vista Internacional de Sociología, tercera época, núm. 1, enero-abril, Madrid, pp. 127 146.

López Jiménez, J. J.; PAniagua Mazorra, A. (1989): «Análisis sociodemográfico del envejecimiento del empresario agrícola en España, III Congreso de Sociología, Sociología Rural, San Sebastián. 
MOPU/ITU (1988): Cambios de la población en el territorio, Dir. F Nasarre, Madrid

NAVArro López, M. (1988): «El contexto sociológico del descenso de la natalidad y el envejecimiento de la población», en Estudios sobre la población del País Valencia, Taula Redonda: Consecuencias socioeconómicas del descenso de la natalidad y envejecimiento de la población, Edicions Alfons el Magnánim, pp 1079-1133.

NAvarro López, M. (1989): La tercera edad en España: aspectos cuantitativos, INSERSO, Servicio de Estadística y Estudios Económicos, nov., núm. 9, Colección Servicios Sociales.

OCDE (1990): Nuevas tendencias en politica agraria, OCDE-MOPU, Madrid.

PANiagua MazorRa, A. (1992): «La población agraria española. Análisis estructural: evolución y perspectivas», Revista Valenciana d'Estudis Autonomics, núm. 14, segunda época, octubre, pp. 247-275, Agricultura Mediterránea, Generalitat Valenciana.

Paniagua MazorRa, A.; LóPez JimÉnez, J. J. (1989): «Rejuvenecimiento versus envejecimiento del empresario agrícola en España: ¿un nuevo comportamiento demográfico en la agricultura española?», en Documentos de Trabajo, CSIC, Madrid, pp. 1-23.

- (1989): «El envejecimiento del empresario agrícola en España», Revista de Estudios Agrosociales, Madrid, pp. 129-158.

Ortells Chabrera, V. (1987): «Cambios recientes en el poblamiento diseminado de las áreas de montaña en el norte del país valenciano (1970-1986)», en I Jornadas de la Población Española, Salamanca, pp. 93-98.

Rodrfguez Rodríguez, V.; Rojo Pérez, F.; Fernández Mayoralas, G. (1986): «Aging of the spanish population (1960-1986), Spatial distribution», Council of Europe' Seminar on demographic problem areas in Europe, Strasbourg, 24 sept.

Rodríguez Rodríguez, V., y Rojo Pérez, F. (1989): «La evolución del envejecimiento en España desde 1900 a 1986: distribución espacial», en II Jornadas sobre la Población Española. Palma de Mallorca, mayo, pp. 381-402.

SÁNCHEZ SÁncheZ, J. (1989): «Áreas de montaña: aproximación a su problemática actual», Espacio, Tiempo y Forma, UNED, Madrid, pp. 169-190.

SAncho Comins, J. (1979-1980): «Contribución al estudio de la población agraria en España», Geographica, Madrid, 227-233.

Vidal Bendito, T. (1987): «La población rural de España. Cambios estructurales 1960 1980», en I Jornadas de la Población Española. Salamanca, pp. 37-49.

ABstract.-Various authors have studied the ageing of the Spanish population and its uneven distribution. While on a national scale, the index of ageing was 0.59 in 1986, implying that $12.18 \%$ of the Spanish population were more than 65 years old, the geographical distribution of the old in the autonomous regions, the provinces and the towns is very uneven. This inequality is even higher when ageing is studied accordig to the size of the nuclei of population. Rural towns which have less than 2,000 inhabitants, according to statistics of the Spanish official body, the I.N.E. in the Census of the Spanish Population, provide figures of an index for the old of higher than unity; indicating that more than one person older than 65 years old for every child younger than 16 years old living in them. If towns with less than 2,000 inhabitants are differentiated into four categories - less than 101, from 101 to 500, from 501 to 1,000 and from 1,001 to 2,000 , it can be observed that the proportion of old people is in inverse proportion to the towns size, and the index is 5.5 in towns with less than 100 inhabitants.

These alarming figures refer to towns which account for only $8.5 \%$ of the Spanish population, but $73.6 \%$ of all towns in Spain, adn more than half the area of the country and make us consider it necessary to carry out a study of these figures with a view to the perspective of the depopulation and abandonment which it implies. 
Resumen.-El envejecimiento de la población española y su desigual distribución espacial es un hecho comprobado y estudiado por diferentes autores. Si bien a escala nacional el índice de envejecimiento JN era de 0,59 en 1986 (el 12,18 de los españoles tenla más de 65 años), la distribución espacial de ese envejecimiento a escalas autonómica, provincial y municipal es muy heterogénea. Esta desigualdad aumenta todavía más cuando se estudia el envejecimiento según el tamaño de los núcleos de población. Los municipios rurales, los que poseen menos de 2.000 habitantes, según propone el INE en el Censo de la Población Española, presentan cifras de envejecimiento por encima de la unidad, es decir, en ellos viven más de un mayor de 65 años por cada niño menor de 15. Diferenciando en cuatro niveles este tamaño poblacional: hasta 101 , de 101 a 500 , de 501 a 1.000 y de 1.001 a 2.000 , se observa que el envejecimiento aumenta en proporción inversa al tamaño de la población de los mismos, siendo del 5,5 en los municipios de menos de $100 \mathrm{~h}$. Estas alarmantes cifras de envejecimiento en una población que, aunque supone tan sólo el $8 \%$ del total de la población española, ocupa el $73,6 \%$ de los municipios y más de la mitad de la superficie total del país, nos hizo pensar en la necesidad de su estudio ante las perspectivas de desertización que ello lleva consigo, 